\title{
Bio-signatures of Planet Earth from Spectropolarimetry
}

\author{
M. F. Sterzik ${ }^{1}$, S. Bagnulo ${ }^{2}$ and C. Emde $^{3}$ \\ ${ }^{1}$ European Southern Observatory, Karl-Schwarzschild-Str 2, D-85748 Garching, Germany \\ email: msterzik@eso.org \\ ${ }^{2}$ Armagh Observatory, College Hill, Armagh BT61 9DG, Northern Ireland, UK \\ ${ }^{3}$ Meteorological Institute, Ludwig-Maximilians-University, Theresienstr. 37, D-80333 Munich, \\ Germany
}

\begin{abstract}
Polarimetry is routinely used to characterise the surfaces of bodies in our solar system. In the near future, polarisation measurements of the starlight reflected by exoplanets will become a common and powerful tool to constrain the atmospheres and the surface properties of other worlds.

If extra-terrestial life has similar signatures as the life we know, then astronomical observations of planet Earth represent a benchmark to eventually probe bio-signatures also on other planets. In fact, linear polarisation spectra of Earthshine (the sunlight that has been first reflected by Earth and then reflected back to Earth by the Moon), allow us to detect the presence of oxygen, ozone, and water in the atmosphere of our planet. Surface properties such as fractional contributions of clouds and ocean, as well as vegetation can be inferred. Ultimately, Earthshine observations provide strong observational constraints on model predictions for Earth-like exoplanets.

In this contribution, we review the most recent observations of Earthshine by polarimetry. We highlight some advances in the interpretation and modelling of whole Earth polarisation, which will be of paramount importance to interpret possible bio-signatures of Earth-like planets in the habitable zone of nearby stars in the future.
\end{abstract}

Keywords. polarisation, radiative transfer, scattering, atmospheric effects, Earth, Moon

\section{Introduction}

Almost 2000 exoplanets orbiting stars other than our Sun have been discovered so far (www.exoplanets.eu), and the quest to find signatures of exo-life has already started. Atmospheres of giant exoplanets are being routinely probed for their composition, and in particular for possible thermochemical disequilibrium constituents (Stevenson et al. 2010). Only recently, the KEPLER mission has revealed statistically robust evidence that Earth-size planets in the habitable zone are commonly found orbiting Sun-like stars (Petigura et al. 2013). Within the next decade we may realistically expect the discovery of planets with rocky cores and masses comparable to the Earth, orbiting in the habitable zone of nearby stars. The detection of bio-signatures in their spectra will be a major goal (Deming \& Seager 2009). Dedicated space missions, and the next generation of extremely large telescopes already under construction should allow to characterise atmospheres of these exoplanets in greater detail.

Until now, planet Earth is the only astronomical object that can be scrutinised for features unique to a life-hosting planet. In a classical experiment, Sagan et al. (1993). used the Galileo spacecraft to record low-resolution spectra of different areas of the Earth. They found molecular oxygen and methane in extreme thermodynamic disequilibrium distributed in the atmosphere, and noticed a sharp increase of the reflectance in the 
red part of the visible spectrum above areas with photosynthetic activity, known as the Vegetation Red Edge (Arnold et al. 2002). Taken together, these features indicate biological activity caused by life as we know it. Our Earth serves as a unique template for the characterisation and interpretation of spectra of to-be-discovered Earth-analogues (Pallé et al. 2009).

\subsection{Polarisation of Earth and Planets}

Light reflected from planets is polarised by scattering processes in the atmosphere or by surface reflection, and it is well known that polarisation spectra can constrain the composition and structure of the planets atmosphere and surface (Coffeen 1979). Polarisation is mainly produced by Rayleigh scattering of molecules or very small aerosol particles in the atmospheres of solar-system planets and is largest near quadrature (Stam 2008). For larger haze and aerosol particles, Mie scattering adds an important contribution to the polarization. Rayleigh (and Mie) scattering is expected to be ubiquitous in exoplanet spectra (Buenzli \& Schmid 2009). The polarisation of hot Jupiters has started to be explored in broad band filters (Berdyugina et al. 2011).

In the case of the Earth or Earth-like planets, polarisation is caused by scattering by the air and by reflection on ocean and land surfaces. The polarised spectrum of the Earth skylight contains signatures of oxygen, ozone, and water. The degree of polarisation of the Earth is a combination of Rayleigh scattering above the ground and cloud tops and depends on the polarising and de-polarising properties of each surface type where light is scattered, refracted, or reflected. Multiple scattering in general randomises the polarisation direction and reduces the degree of polarisation. Fine-grained surfaces (regolith scattering) cause an inverse correlation between albedo and polarisation degree at large phase-angles (Umov effect, Zubko et al. 2011). Similarly, optically thick clouds depolarise through multiple scattering. In molecular absorption bands, light is scattered less and has a higher degree of polarisation, and thus shows up in emission; this has been detected from the ground for the Earth's atmosphere (Aben et al. 1999) and for gas giants (Joos \& Schmid 2007). Tropospheric aerosol particles impact the polarisation of skylight, and in particular the polarisation in the $\mathrm{O}_{2}-\mathrm{A}$ band $(760 \mathrm{~nm})$ represents an important diagnostic to derive their microphysical properties. Depending on the stratification of the atmosphere, mixing ratios of the gaseous components and the altitude of the cloud decks can be derived (Boesche et al. 2008).

On Earth, at angles of around $140^{\circ}$, scattering by cloud droplets gives rise to a rainbow, with a relatively high fractional polarisation. Detection of this rainbow allows one to determine the thermodynamical phase in a cloud (Goloub et al. 2000). The fractional polarisation of the disk-integrated rainbow peak for Earth has been estimated to be around $15 \%$ and has been proposed to be observable also for exoplanets (Bailey 2007). In addition, stellar light that is specularly reflected by surface oceans may become prominently polarised and observable for exoplanets (Williams \& Gaidos 2008).

The greatest technical challenge for observations of exoplanets is the enormous flux difference between the overwhelmingly bright star and the reflected light of a spatially hardly-resolved planet. Polarimetry, and in particular spectropolarimetry, may help to enhance their contrast because the polarisation of the stellar radiation and of the interstellar medium is small and featureless, whereas the polarisation caused by scattering in a planetary atmosphere is high, and rich in spectral features. The polarised part can be differentiated from the unpolarised component of the radiation, and permits to disentangle the planet contribution from the observed spectrum. Since the radiation of star and planet are recorded simultaneously and through the same optical path on the detector, polarimetric techniques do not need absolute flux calibrations, and are largely 


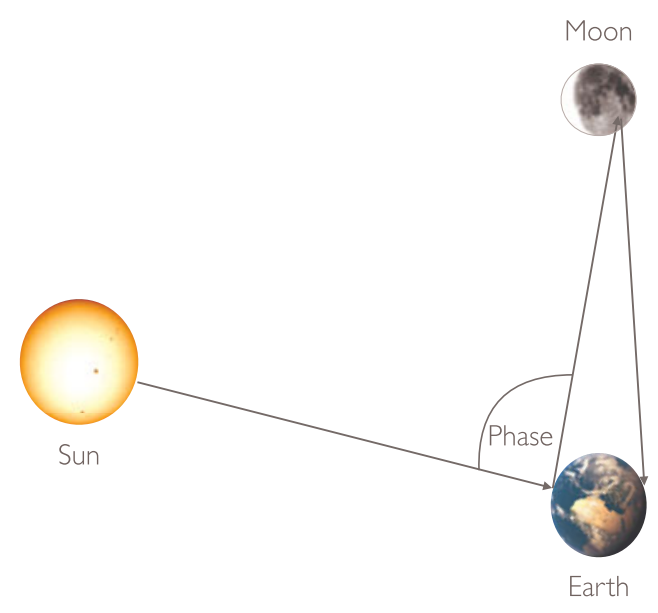

Figure 1. Sketch of the geometry for Earthshine observations (not to scale). The phase-angle $\alpha$ is defined between Sun, Earth and the Moon. The light is diffusly scattered back from the lunar surface to Earth. The scattering angle on the lunar surface is about one degree only.

insensitive to the Earth's atmospheric conditions, and to stellar variation. Polarimetry is thus a particularly powerful diagnostic tool for ground-based applications.

\subsection{Earthshine}

Observations of Earthshine allow us to measure the reflected light of whole Earth from ground. The Earthshine is the sunlight scattered by the dayside Earth, reflected back to Earth from the darker portion of the visible Moon. This fraction of the lunar surface acts as a diffuse reflector that spatially integrates the light coming from the sunlit side of the Earth. Different surface areas of the Earth can be probed as the relative SunEarth-Moon viewing geometry changes (phase-angle $\alpha$ ). Thus, the Earthshine allows one to perform ground-based observations of the disk-integrated Earth as seen from space (see sketch in Fig.1). Bio-signatures have already been detected in Earthshine intensity spectra. However, their interpretation is ambiguous, and depends critically on model assumptions such as the Earths albedo at a given observing epoch (Seager et al. 2005 and Montanes-Rodriguez et al. 2006).

\subsection{First polarisation measurements of whole Earth}

Surprisingly only a few, and mostly broadband, polarimetric measurements of the Earth had been obtained in the past. Classical observations of Earthshine at a wavelength of $550 \mathrm{~nm}$ report a fraction of linear polarization of about $10 \%$ near a phase angle of $90^{\circ}$ (Dollfus 1957). Since the lunar surface is responsible for a depolarization of the radiation by a factor of $\approx 3$, the true linear polarization of the globally integrated light of the Earth was estimated to be around 30-35\%. Modern estimates of the whole-Earth polarization are based on the satellite borne instrument POLDER. In three bands $(443 \mathrm{~nm}, 670 \mathrm{~nm}$ and $865 \mathrm{~nm}$ ) polarisation levels between $4 \%-40 \%$ are being inferred, the large spread caused by a strong dependence on cloud cover, and on wavelength (Wolstencroft \& Breon 2005).

\section{Spectro-polarimetric observations of Earthshine}

In the following we describe the most recent measurements of whole Earth polarisation observed through Earthshine. 

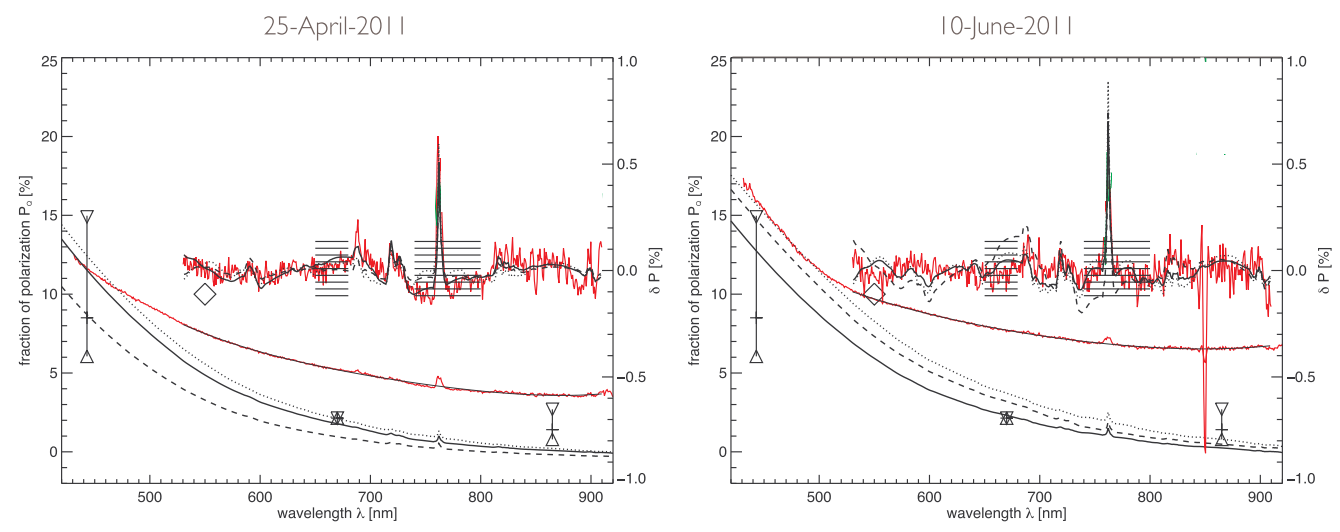

Figure 2. Linear optical polarisation spectra of Earthshine as observed with the VLT at two different epochs. The scale of the spectra is different for the continuum and for the continuum subtracted residuals. Model spectra are plotted with thick lines (different line styles indicate different model parameters), observations with thin lines (red in the electronic version).

\subsection{Biosignatures revealed by FORS at the VLT}

Linear polarisation spectra of Earthshine were obtained and interpreted for the first time by Sterzik et al. (2012). They observed Earthshine with the FORS2 instrument mounted at the ESO Very Large Telescope located in Chile. The useful spectral range covered a wavelength region between 450 and $920 \mathrm{~nm}$ with a resolution element of about $3 \mathrm{~nm}$. Two distinct viewing geometries of Earth were observed near quadrature (phase-angle $\alpha$ near 90 ). During the first observing epoch (dawn on April 25 2011), Earthshine contained contributions from the Atlantic sea, the Amazonas, and parts of Europe and Africa, and the visible surface consists of $72 \%$ clouds, $18 \%$ ocean, about $7 \%$ vegetated, and minor fractions of un-vegetated areas, retrieved from MODIS satellite data. The second observing epoch (dusk on June 9 2011) probed the Pacific side of Earth (50\% clouds and $46 \%$ ocean) with almost no land surface areas visible $(<5 \%)$. The spectra allow to discern the fractional contribution of clouds and ocean surface, and diagnose small amounts of vegetated areas when compared with theoretical vector radiate transfer models of Stam (2008).

The curves in Fig.2 (in the electronic version in red colour) display the fraction of linear polarisation $P_{Q}(\lambda)$ measured in April (left panel) and in June (right panel). The shape of the $P_{Q}(\lambda)$ profile depends on Earths surface albedo at the time of observations. In April, the continuum polarisation reaches about $9 \%$ at $500 \mathrm{~nm}$ and decreases smoothly to $4 \%$ at $900 \mathrm{~nm}$. The polarisation observed in June is $3 \%$ higher than observed in April (full scale axis to the left).

Note that the Earthshine spectra are reflected back on the lunar surface, which depolarises. This depolarisation on the lunar surface depends on wavelength, but reliable models are not available. For the scaling of the models to the Earthshine, depolarisation according to Fox et al. (1998) was assumed which is approximately a factor of 3 and dependant on wavelength.

Thick black curves in Fig. 2 indicate model spectra of Stam (2008) that are designed to resemble the geometry and surface structure during the observations. The model parameters that fit the observations best are plausible approximations to the real surface fractions responsible for the Earthshine spectrum at the time of the observations. The slope of the models are steeper than the observations and are, in particular in the red part of the spectrum, too low for all reasonable assumptions of cloud coverage. On the 
other hand, in the blue part of the spectrum (between 420 and $530 \mathrm{~nm}$ ) the best models retrieve cloud and ocean surface coverages that closely resemble the satellite retrieval. The smaller polarisation observed in April can be well explained by the approximately $20 \%$ higher cloud coverage.

The detailed spectral structure is visible only after subtracting the continuum from the observed $P_{Q}(\lambda)$ profile (fitting a fourth-order polynomial between 490 and $910 \mathrm{~nm}$ ). The residual signal $\delta P$ shows a rich structure at the $0.1 \%$ level (right axis scale). A narrow $\mathrm{O}_{2}$-A band is prominent in both spectra at the level of almost $1 \%$ above the continuum. $\mathrm{O}_{2}-\mathrm{B}(690 \mathrm{~nm})$ and $\mathrm{H}_{2} \mathrm{O}(720$ and $820 \mathrm{~nm})$ bands are broader and with lower amplitude. An additional narrow polarisation feature is present in the June spectrum at $850 \mathrm{~nm}$, which can be attributed to an instrumental artefact (internal reflection). Most remarkably, however, the overall shape of the residuals between 550 and $750 \mathrm{~nm}$ differs in the two epochs: while the April spectrum exhibits a broad shallow peak around 700nm, the June spectrum appears flat.

The residual model spectra explain this difference. The residual profiles are generated in the same way as the observed ones by subtracting the continuum: in general, they appear to be fully consistent with the observed spectra. Two bandpass regions at $650-680 \mathrm{~nm}$ and $740-800 \mathrm{~nm}$ represent regions for which the normalised difference vegetation index can be calculated (NDVI, Tinetti 2006). A larger contrast in the continuum averages in April than in June can be interpreted by the presence of a Vegetation Red Edge signal in April, which is practically not detected in June. The Vegetation Red Edge feature detected in the April spectra can be induced by assuming a fraction of 5-10\% cloud-free landvegetation in the models. Contrary, the June data can best be described through little or absent cloud-free vegetation surfaces. The observed polarisation peak of the $\mathrm{O}_{2}$-A feature (and to a lesser extend $\mathrm{O}_{2}-\mathrm{B}$ ) is also compatible with the models. For a given spectral resolution, the amplitude of these features are sensitive to the top of the cloud layer, and to the $\mathrm{O}_{2}$ mixing ratio.

\subsection{Phase dependance of the Broadband Earthshine Polarisation}

Bazzon et al. (2013) measured the fractional polarisation of Earthshine $P_{\mathrm{es}}$ in the $B V R I$ filters for Earth phase-angles $\alpha$ between 30 and 110 degree with a new, specially designed wide field polarimeter. Their data were taken in March and October 2011. In their observations, the light from the bright lunar crescent is blocked with focal plane masks. Because the entire Moon is imaged, the Earthshine observations can be corrected for the stray light from the bright lunar crescent and twilight. They can fit the phase dependence by a function $P_{\mathrm{es}}=q_{\mathrm{max}} \sin ^{2} \alpha$, as expected. They introduce an improved lunar depolarisation methodology, and derive the lunar surface polarisation efficiency depending on wavelength $\lambda$ and the lunar surface albedo $a$ using measurements of lunar samples from the literature. They find that polarisation of Earthshine decreases toward longer wavelengths and is about a factor 1.3 lower for the higher albedo highlands. For mare regions they measure a maximum polarisation of about $q_{\max , \mathrm{B}}=13 \%$ for $\alpha=90^{\circ}$ (half moon) in the $B$ band. Taking into account the depolarisation, the resulting fractional polarisations for the planet Earth derived from their Earthshine measurements are 24.6\% for the $B$ band, $19.1 \%$ for the $V$ band, $13.5 \%$ for the $R$ band, and $8.3 \%$ for the $I$ band. Together with the literature values for the spectral reflectivity, they obtain a contrast between the polarised flux of the planet Earth and the (total) flux of the Sun with an uncertainty of less than $20 \%$, and find that the best phase for detecting an Earth twin will be around $\alpha \approx 65^{\circ}$. Their results are very useful to assess different instrument and observing strategies for future high contrast polarimetry of extrasolar planetary systems. 
The relatively high continuum polarisation of planet Earth for red optical wavelengths is corroborated.

\subsection{Phase dependance of Earthshine Polarisation Spectra}

Takahashi et al. (2013) present more results of optical spectropolarimetry of Earthshine for phase angles ranging from $49^{\circ}$ to $96^{\circ}$. They report observations conducted on 2011 March 9-13 (UT) using the spectropolarimeter HBS installed on the $1.88 \mathrm{~m}$ telescope at Okayama Astrophysical Observatory, covering wavelengths between 450 and $850 \mathrm{~nm}$ with a spectral resolution of $6 \mathrm{~nm}$. Again, the degree of Earthshine polarisation decreases with increasing wavelength at any phase, while the overall degree of polarisation increases as the Earth approaches quadrature. They find that the phase dependence differs with the wavelength: the maximum polarisation for the $V$ band occurs at a phase-angle of $90^{\circ}$, whereas for longer wavelengths it is shifted towards larger phase angles. They interpret their result indicating that Earthshine polarisation at shorter wavelengths is dominated by atmospheric Rayleigh scattering, whereas it exhibits an increasingly effective contribution from the Earth surface reflection at longer wavelengths. They suggest that the wavelength dependence of the phase-angle of the maximum polarisation is a distinctive characteristic of Earth: a planet that has a scattering, but transparent atmosphere above its surface.

\subsection{Near-infrared Earthshine Polarisation Spectra}

Miles-Paez et al. (2014) extend the integrated planet Earth spectropolarimetry from optical to near-infrared wavelengths. Major biomarkers like $\mathrm{O}_{2}$ and water vapor are strong flux absorbents in the Earths atmosphere, and some linear polarization of the reflected stellar light is expected to occur at these wavelengths. They present simultaneous optical (400-900nm) and near-infrared $(0.9-2.3 \mu \mathrm{m})$ linear polarisation spectra of Earthshine, observed on 2013, May 18 with the instrument ALFOSC on the Nordic Optical Telescope and LIRIS at the William Herschel Telescope, both situated on La Palma. The spectral resolution $(2.5 \mathrm{~nm}$ in the optical, and 1.8 and $2.9 \mathrm{~nm}$ in the near-infrared) suffices to resolve major molecular species present in the Earth's atmosphere. Consistent with all observations previously reported above, they find the highest values of linear polarization $(\geqslant 10 \%)$ at the bluest wavelengths. Linear polarisation steadily decreases toward red wavelengths reaching a nearly flat value beyond 800nm. Interestingly, they measure a relatively high polarisation degree of $\approx 4.5 \%$ for the continuum in the near-infrared. They detect molecular features due to $\mathrm{O}_{2}$ at $0.760,1.25 \mu \mathrm{m}$, and $\mathrm{H}_{2} \mathrm{O}$ at $0.653-0.725 \mu \mathrm{m}$, $0.780-0.825 \mu \mathrm{m}, 0.93$, and $1.12 \mu \mathrm{m}$, most of them having a high linear polarisation degree above the continuum. Most notably and unexpectedly, the broad $\mathrm{H}_{2} \mathrm{O} 1.12 \mu \mathrm{m}$ band displays a polarisatoin degree as high as $10 \%$.

\section{Advances in Earth polarisation modelling}

The original polarisation model spectra of Earth by Stam (2008) are based on an adding-doubling one dimensional vector radiative transfer algorithm, which fully includes multiple scattering and polarisation. The atmospheres of the model planets are described by stacks of homogeneous layers containing gaseous molecules and, optionally, cloud particles. Each model atmosphere is bounded below by a flat, homogeneous surface. Horizontally inhomogeneous planets are approximated by weighting results obtained for homogeneous surfaces. Composition, structure, and optical properties of the model atmospheres and the reflection properties of the model surfaces implemented are mostly 


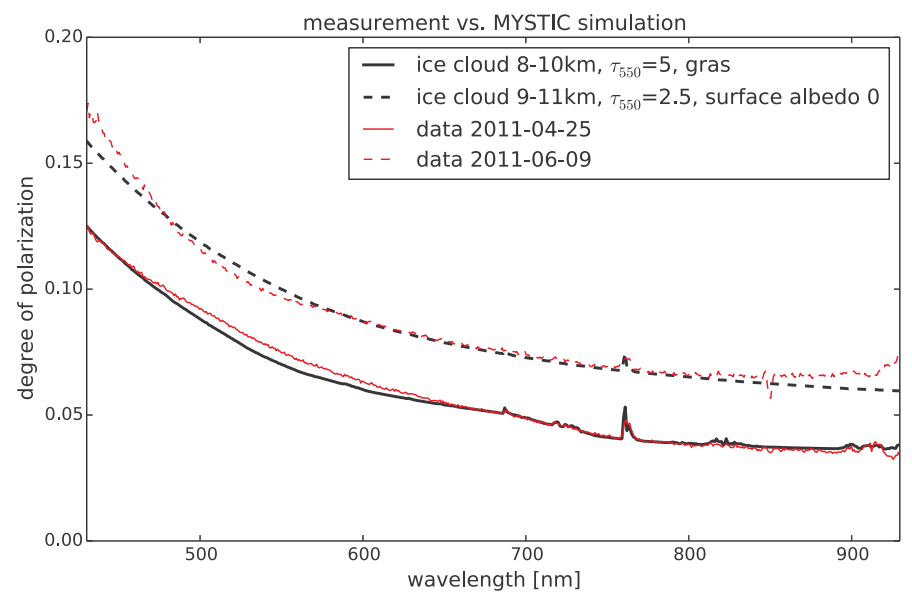

Figure 3. Comparison of two observed Earthshine spectra (from Sterzik et al. 2012) as shown in Fig. 2 with Monte Carlo simulations based on the methodology described in Emde et al. (2010). Shown are observed spectra (thin lines - red ) and model spectra (thick lines - black).

simplifications. Overall, these models already describe the observed spectra, and in particular the narrow line features caused by atmospheric absorption and scattering with a surprisingly high degree of fidelity and accuracy. All Earthshine observations, however, yield a much higher degree of continuum polarisation in the red part of the spectrum (up to a factor 3 to 5 ), which remains to be explained.

The models are very sensitive to the average cloud properties integrated over whole Earth as their optical properties mainly affect the overall albedo, scattering and polarisation properties at large. Several advances with respect to the original models have been made meanwhile. Karalidi et al. (2011) consider liquid water clouds with different optical thicknesses, altitudes, and particle sizes, as functions of the phase-angle $\alpha$ to calculate flux and polarisation spectra of Earth-like planets. They find that shapes and strengths of polarisation features depend strongly on the cloud particles size and shape, and can thus be used for particle characterisation. Horizontally inhomogenuous planetary surfaces are considered in Karalidi \& Stam (2012). They find that the shape of the polarisation phase function is sensitive to horizontal inhomogeneities and to patchy clouds above an ocean or a sandy continent of an Earth-like planet seen in integrated light.

A different approach to model the polarisation spectra of Earth is followed by Emde et al. (2010). They use a full three dimensional Monte Carlo vector radiative transport code MYSTIC which allows to simulate curved geometries and inhomogeneous planets. An efficient numerical sampling method permits broad spectral coverage and high spectral resolution (Emde et al. 2011).

Aerosols, and high ice clouds are important and realistic constituents of Earths atmosphere, which had so far not been taken into account in the models. As an example, the high sensitivity of Earths polarisation on the introduction of high ice clouds is demonstrated in Figure 3. It compares the Earthshine polarisation spectra obtained in April and June 2011 from Sterzik et al. (2012) with new model calculations. Gas absorption has been included using the REPTRAN parameterisation (Gasteiger et al. 2014). We used the HEY parameterisation for ice crystal optical properties as included in the libRadtran package (www.libradtran.org) and assume that the crystals are solid columns with an "effective radius" of $30 \mu \mathrm{m}$. The solid line shows a simulation for an ice cloud layer from 8 to $10 \mathrm{~km}$ with a cloud optical thickness at $550 \mathrm{~nm}$ of 5 . The underlying spectral surface 
albedo for gras is taken from measurements by Feister \& Grewe (1995). The dashed line is for an ice cloud layer with an optical thickness at $550 \mathrm{~nm}$ of 2.5 in 9 to $11 \mathrm{~km}$ altitude above a black surface. These simulations in comparison to the measurements show that ice clouds may be the key to explain the high degree of continuum polarisation in the red part of the spectrum.

More systematic comparisons of state-of-the-art models using realistic assumptions about the most relevant mechanisms that determine the polarisation state of whole Earth and observations with highly accurate determinations of the polarisation degree are needed to further constrain the expected signatures of exo-Earths.

\section{References}

Aben, I., Helderman, F., Stam, D. M., \& Stammes, P. 1999, Geophys. Res. Let. 26, 591

Arnold, L., Gillet, S., Lardiere, O., Riaud, P., \& Schneider, J. 2002, A\&A 392, 231

Bailey, J. 2007, Astrobiology 7, 320

Bazzon, A., Schmid, H. M., \& Gisler, D. 2013, A $\& A$ 556, A117

Berdyugina, S. V., Berdyugin, A. V., Fluri, D. M., \& Piirola, V. 2011, Ap. Lett. 728, L6

Boesche, E., Stammes, P., Preusker, R., Bennartz, R., Knap, W., \& Fischer, J. 2008, App. Optics 47,3467

Buenzli, E. \& Schmidt, H. M. 2009, A\&A 504, 259

Coffeen, D. L. 1979, J.Opt.Soc.Am. 69, 1051

Deming, D. \& Seager, S. 2009, Nature 462, 301

Dollfus, A. 1957, Suppl. aux Annales d'Astrophysique 4, 58

Emde, C., Buras, R., Mayer, B., \& Blumthaler, M. 2010, Atmos. Chem. Phys. 10, 383

Emde, C., Buras, R., \& Mayer, B. 2011, J. Quant. Spectrosc. Radiat. Transfer 112, 1551

Feister, U. \& Grewe, R. 1995, Photochem. Photobiol. 62, 736

Fox, G. K., et al. 1998 MNRAS 298, 303

J. Gasteiger, Emde C., Mayer B., Buras R., Buehler S. A., Lemke O. 2014, J. Quant. Spectrosc. Radiat. Transfer, 148, 99

Goloub, P., Herman, M., Chepfer, H., Riedi, G., Brogniez, G., Couvert, P., \& Seze, J. 2000 Journal of Geophys. Res. 105, 14747

Joos, F., \& Schmid, H. M. 2007 A\& A 463, 1201

Karalidi, T., \& Stam, D. M. 2012 A $\& A$ 546, A56

Karalidi, T., Stam, D. M., \& Hovenier, J. W. 2011 A $\& A$ 530, A69

Miles-Páez, P. A., Pallé, E., \& Zapatero Osorio, M. R. 2014, Astron. Lett. 562, L5

Montanes-Rodriguez, P., Palle, E., Goode, P. R., \& Martin-Torres, F. J. 2006, ApJ 651, 544

Pallé, E., Zapaerto Osorio, M. R., Barrena, R., Montanes-Rodriguez, P., \& Martin, E. L. 2009, Nature 459, 814

Petigura, E. A., Howard, A. W., \& Marcy, G. W. 2013, PNAS 110, 19273

Sagan, C., Thompson, W. R., Carlson, R., Gurnett, D., \& Hord, C. 1993, Nature 365, 715

Seager, S., Turner, E. L., Schafer, J., \& Ford, E. B. 2005, Astrobiology 5, 372

Stam, D. 2008, A\&SA 482, 989

Sterzik, M. F., Bagnulo, S., \& Pallé, E. 2012, Nature 483, 64

Stevenson, K. B., Harrington, J., Nymeyer, S., Madhusudhan, N., Seager, S., Bowman, W. C., Hardy, R. A., Deming, D., Rauscher, E., \& Lust, N. B. 2010, Nature 464, 1161

Takahashi, J., Itoh, Y., Akitaya, H., Okazaki, A., Kawabata, K., Oasa, Y., \& Isogai, M. 2013, PASJ 65, 38

Tinetti, G., et al. 2006, Astrobiology 6, 881

Williams, D. M. \& Gaidos, E. 2008, Icarus 195, 927

Wolstencroft, R. \& Breon, F.-M. 2005, in: A. Adamson, C. Aspin, C. J. Davis, \& T. Fujiyoshi (eds.), Astronomical Polarimetry: Current Status and Future Directions, ASP Conf. Series 343 (San Francisco: ASP), p. 211

Zubko, E., Videen, G., Shkuratov, Y., Muinonen, K., \& Yamamoto, T. 2011, Icarus 212, 403 